\title{
Microwave Oscillator Ultrasound Receivers
}

\author{
Tom S Robbins*, James P Seddon*, Erwin J Alles ${ }^{\dagger}$, \\ Edward Z Zhang ${ }^{\dagger}$, Adrien E Desjardins ${ }^{\dagger}$ and Cyril C Renaud* \\ * Department of Electronic and Electrical Engineering, \\ University College London \\ London, United Kingdom \\ Email: thomas.robbins.15@ucl.ac.uk \\ $\dagger$ Department of Medical Physics and Biomedical Engineering, \\ University College London, \\ London, United Kingdom
}

\begin{abstract}
We propose and demonstrate a miniature microwave device capable of detecting wide-band ultrasound without the use of piezo materials. By the nature of the transduction mechanism, the scheme naturally implements orthogonal frequency division multiplexing (OFDM): each device can be assigned its own microwave band, mitigating electrical cross-talk between adjacent sensor elements for future imaging array systems. Furthermore, the entire array could be addressed with a single electrical connection, since addressing is in the frequency, rather than spatial, domain. We anticipate that the convenience afforded by this fully electronic platform will enable its use as a low cost and versatile solution in cases where high sensitivity is required over a large portion of the acoustic spectrum.

Index Terms - ultrasound, microwaves, sensors, RF
\end{abstract}

\section{Introduction}

Ultrasound imaging techniques which use short, broadband pulses - such as laser generated ultrasound [1] and photoacoustics [2] - require receivers which are sensitive across a wide acoustic spectrum [3]. Currently, piezoceramic transducers are often used in these applications: due to the high acoustic impedance of piezoceramics, they resonate according to their machined dimensions when coupled to low Young's modulus media such as biological tissue or water. However, this makes them highly sensitive only to signals around their resonant frequency. Wideband receivers such as PVDF hydrophones, which feature piezo crystals embedded in a polymer matrix, offer better broadband performance, although at the expense of peak sensitivity [4]. Furthermore, scaling down elements made from these materials in order to increase their acoustic angle of acceptance and allow higher resolution field sampling causes a corresponding reduction in sensitivity [5], limiting their performance for high contrast imaging.

We propose a new type of electronic ultrasound receiver whose operation is inspired by the interferometric techniques used in optical ultrasound sensors [6], but implemented in the microwave frequency band instead

This work was supported by EPSRC Centre for Doctoral Training (CDT) in Integrated Photonic and Electronic Systems (IPES) $(\mathrm{EP} / \mathrm{L} 015455 / 1)$ of the near infra-red or visible. Unlike capacitive micromachined ultrasound transducers, it requires no MEMS fabrication processes to produce [7], and the flexibility offered by its simple construction makes it possible to incorporate acoustic lensing, as commonly found in piezo transducers. At the same time, it benefits from all the advantages of a fully electronic sensor, such as low cost, low power and easy multiplexing. Since each sensing element can carry the ultrasound signal on its own microwave band, the electrical cross-talk inherent in other arrays is eliminated [8]. Finally, since the sensing mechanism is based on an electromagnetic resonance, the effective length of interaction between the ultrasound field and the sensor is scale-invariant, meaning the element size can be reduced without loss of sensitivity. We present here some preliminary results from a prototype single-element device developed and manufactured entirely within our own labs. We demonstrate successful detection of a $5 \mathrm{MHz}$ ultrasound pulse, estimate the device sensitivity, and describe potential areas for improvement.

\section{Theory and Principle}

The device comprises a pressure-sensitive microwave resonator, depicted schematically in Figure 1. It operates in analogy to the Fabry-Perot optical devices, only instead of the phase change being produced by an optical cavity which changes in length [3], we have a microwave resonator whose resonant frequency is perturbed by pressure changes. In either case, the resonance shifts, the corresponding amplitude envelope can be detected, and from that an ultrasound signal measured.

The resonator is excited near its fundamental mode, at frequency $\omega_{0}$. As the boundary conditions of the resonator are changed, the amplitude of the signal is modulated. For an exemplary $1 \mathrm{D}$ system, this can be represented by

$$
E=A \cos \left(\omega_{0} t\right)
$$

where $E$ is the electric field component of the microwave oscillation, $\omega_{0}$ is its angular frequency, and $t$ is time. The amplitude $A$ is modulated by the ultrasound signal with frequency $\omega_{u}$ and amplitude $A_{u}$, giving 


$$
\begin{aligned}
E & =A_{u} \cos \left(\omega_{u} t\right) \cos \left(\omega_{0} t\right) \\
& =A_{u} \cos \left[\left(\omega_{0}+\omega_{u}\right) t\right] / 2+A_{u} \cos \left[\left(\omega_{0}-\omega_{u}\right) t\right] / 2 .
\end{aligned}
$$

I.e., the ultrasound signal can be detected on the sidebands of the high-frequency signal at $\omega_{0}+\omega_{u}$ and $\omega_{0}-\omega_{u}$. For a broadband ultrasound pulse, the signal would be represented by the summation of the continuum of frequencies $\left\{\omega_{u}\right\}$ with amplitudes given by their Fourier components $\left\{A_{u}\right\}$.

\section{Methods}

We aimed to create a device with a sub- $3 \mathrm{GHz}$ resonance in as small a footprint as possible, allowing it to be operated using widely available microwave sources whilst having a small acoustic aperture. A prototype singleelement device with a resonance around $1.8 \mathrm{GHz}$ was designed and simulated using the CST Microwave Studio software package. After fabrication - details of which are omitted here due to intellectual property constraintsthe resonance was characterised using a network analyser (EC5071C ENA Series Network Analyser, Aligent Technologies) to measure the transmission $\left(S_{21}\right)$ and reflection $\left(S_{11}\right)$ spectra. The position of the resonance was found to be within reasonable error of the FDTD simulation (within $2 \%$ ). After this initial characterisation, the device was set up in the configuration illustrated by Figure 2. To enhance the sensitivity, a homodyne detector was implemented using a power splitter (ANRITSU K241B), phase shifter (ARRA 9426R) and frequency mixer (Mini-Circuits ZX05$\mathrm{C} 42$ ). The output from this was passed through a lowpass filter (passband from $\mathrm{DC}$ to $80 \mathrm{MHz}$ ) and then an

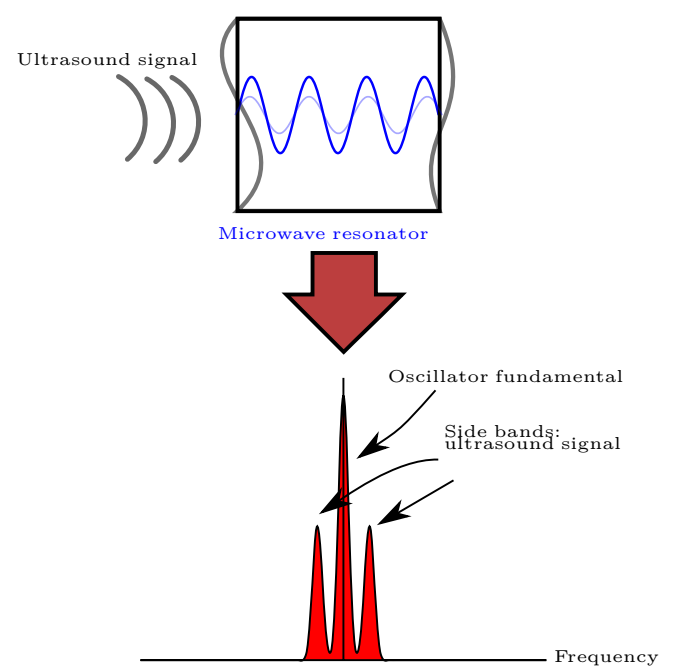

Fig. 1. The microwave resonator, represented by a box, is perturbed by an ultrasound signal. The fundamental mode of the resonator, represented by the blue standing wave in the box and the large central peak in the spectrum, is modulated by the changing boundary conditions of the resonator, resulting in the depicted sidebands which carry the ultrasound signal. ultrasound receiver with a high gain amplifier (Olympus 5072PR). We then coupled a piezo ultrasound transducer (Harisonic 13-0504-R) to the top side of the device. A block of pure gel wax [9] was used as a spacer between the device and the transducer. The piezo transducer was driven by a train of pulses at its resonant frequency $(5 \mathrm{MHz})$ from an ultrasound pulse generator and receiver (Olympus 5077PR).

A high-frequency microwave source (Keysight N9310A RF Signal Generator) was tuned to near the quadrature (point of greatest slope) of the device's electromagnetic resonance and connected to the system. This tuning was achieved by running the signal from the device through a power detector (Pasternack PE8014) and taking voltage readings whilst changing the frequency of the RF source. To detect ultrasound, an oscilloscope (Agilent Technologies DSO-X 3024A) was externally triggered by the ultrasound pulse generator and traces for both the transducer and receiver were displayed. Since the ultrasound pulse reflects off the sensor back to the transducer, we observe two pulses generated by the pulse transceiver: an initial pulse representing the ultrasound being generated at the transducer interface at time $t=0$; and a second pulse after the time taken to make a round trip, $t=T$. Hence the response of the device can be seen as the pulse on the second trace half-way between the two pulses from the transceiver, at time $t=T / 2$ (see Figure 3). Pressing the transducer closer to the receiver and deforming the gel wax block changes the ultrasound path length $T$. As a final check, we confirmed that even when displacing the transducer in this way, the signal at the receiver did indeed remain at $T / 2$. In this way we eliminated any doubt regarding the origin of the electrical signal from the receiver.

\section{Results and Discussion}

The transmission spectrum of the device is shown in Figure 4. The device simulations predict a resonance Qfactor of 8000 . However, in practice, manufacturing defects and material losses result in a measured Q-factor of 40 . The homodyne configuration shown in Figure 2 enhances the sensitivity of the system, although at the expense of increased signal loss.

From a semi-empirical model, we determined the sensitivity of the resonance to perturbations of its boundary conditions to be $0.57 \mathrm{MHz} / \mu \mathrm{m}$. By varying the frequency of the RF source and measuring the output voltage, we found the system had a peak sensitivity of $0.6 \mathrm{mV} / \mathrm{MHz}$ at the point of largest gradient near the microwave resonance of the device. Using a model derived from [3], the deformation due to ultrasound was calculated as $0.2 \mu \mathrm{m} / \mathrm{MPa}$. Multiplying these three figures gives an estimate for the device sensitivity: $0.07 \mathrm{mV} / \mathrm{MPa}$. The noise of the system, including the $60 \mathrm{~dB}$ amplifier, was measured experimentally as $390 \mathrm{mV} / \sqrt{200 \mathrm{MHz}}$ peak-to-peak noise, 


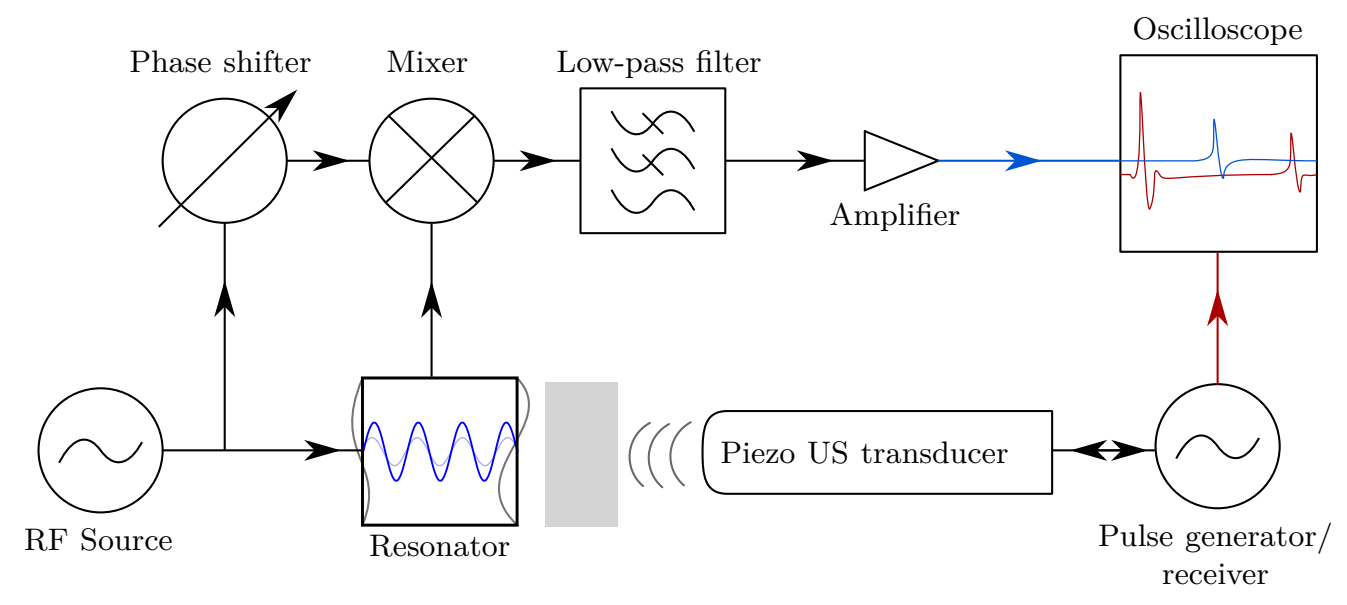

Fig. 2. Block diagram of the system for testing the ultrasound receiver

therefore we estimate the noise equivalent pressure (NEP) detectable by our system to be $5.7 \mathrm{kPa} \sqrt{200 \mathrm{MHz}}$.

In practice, we were able to observe $5 \mathrm{MHz}$ pulses generated by the piezo transducer driven at $400 \mathrm{~V}_{\mathrm{p}-\mathrm{p}}$ at an SNR of about 2 (over a $200 \mathrm{MHz}$ measurement bandwidth, see Figure 5). Some degradation of the signal can be accounted for by the large losses introduced by the homodyne system, and also in acoustic losses not accounted for in our model. Sources of noise in the system include background electromagnetic interference, phase noise from the RF source, and noise introduced by the amplifier and mixer.

Integrating the sensing system onto a single PCB would reduce noise and losses by removing most of the RF coaxial connections and shortening transmission lines. It would also significantly reduce the cost and size of the system. Once integrated in such a way, it would be possible to produce a compact array of sensing elements, with minimal electrical cross-talk, and a single electrical lead and readout pathway. In the present design, the sensing element footprint is about $13 \mathrm{~mm}^{2}$. However, with a ten-fold increase in microwave frequency (still within the widely attainable frequency band of sub-20 GHz) and hence tenfold reduction in length-scale, the footprint reduces to under $1 \mathrm{~mm}^{2}$. Further improvements to the resonator design could bring this value even lower. Finally, we note that the fabrication method of the receivers could potentially be adapted for non-planar or even flexible arrays or elements, enabling various acoustic lensing possibilities.

\section{Conclusion}

An EM-resonance-based microwave ultrasound detector could enable wide-band ultrasound detection at sensitivities not possible with existing PVDF sensors. For a future imaging system, the possibility of assigning each sensing element its own RF band could result in reduced electrical cross-talk between sensing elements. With improvements, such as replacing lab-bench equipment with integrated circuits, noise equivalent pressure ratings below $400 \mathrm{~Pa} \sqrt{\mathrm{MHz}}$ could be realised.

\section{Acknowledgements}

TSR thanks Efthymios Maneas for supplying the gelwax material, and James Guggenheim and Paul Beard for providing helpful discussions and encouragement. He also thanks Martin Scott and John Langdon for their technical contributions.

\section{References}

[1] R. J. Colchester, C. A. Mosse, D. S. Bhachu, J. C. Bear, C. J. Carmalt, I. P. Parkin, B. E. Treeby, I. Papakonstantinou, and A. E. Desjardins, "Lasergenerated ultrasound with optical fibres using functionalised carbon nanotube composite coatings," Applied Physics Letters, vol. 104, no. 17, p. 173502 , Apr. 2014, issn: 0003-6951, 1077-3118. doi: 10.1063/1. 4873678. [Online]. Available: http://scitation.aip.org/ content/aip/journal/apl/104/17/10.1063/1.4873678 (visited on 11/20/2015).

[2] P. Beard, "Biomedical photoacoustic imaging," en, Interface Focus, vol. 1, no. 4, pp. 602-631, Aug. 2011, issn: 2042-8898, 2042-8901. doi: $10.1098 /$ rsfs . 2011 . 0028. [Online]. Available: http : / / rsfs . royalsocietypublishing.org/content/1/4/602 (visited on $12 / 16 / 2015)$.

[3] P. C. Beard and T. N. Mills, "Extrinsic optical-fiber ultrasound sensor using a thin polymer film as a low-finesse Fabry-Perot interferometer," EN, Applied Optics, vol. 35, no. 4, pp. 663-675, Feb. 1996, issn: 2155-3165. doi: 10.1364/AO . 35 . 000663. [Online]. Available: https://www.osapublishing.org/abstract. cfm?uri=ao-35-4-663 (visited on 12/15/2017). 
[4] M. D. Sherar and F. S. Foster, "The design and fabrication of high frequency poly(vinylidene fluoride) transducers," Ultrasonic Imaging, vol. 11, no. 2, pp. 75-94, Apr. 1989, issn: 0161-7346. doi: 10.1016/ 0161 - 7346(89 ) 90001 - 1. [Online]. Available: http : / / www . sciencedirect . com / science / article / pii / 0161734689900011 (visited on 02/26/2018).

[5] J. A. Guggenheim, J. Li, T. J. Allen, R. J. Colchester, S. Noimark, O. Ogunlade, I. P. Parkin, I. Papakonstantinou, A. E. Desjardins, E. Z. Zhang, and P. C. Beard, "Ultrasensitive plano-concave optical microresonators for ultrasound sensing," En, Nature Photonics, vol. 11, no. 11, p. 714, Oct. 2017, issn: 1749-4893. doi: 10.1038/s41566-017-0027-x. [Online]. Available: https://www.nature.com/articles/s41566017-0027-x (visited on 11/03/2017).

[6] P. C. Beard and T. N. Mills, "Miniature optical fibre ultrasonic hydrophone using a Fabry-Perot polymer film interferometer," Electronics Letters, vol. 33, no. 9, pp. 801-803, Apr. 1997, issn: 0013-5194. doi: 10.1049/el:19970545.

[7] A. Caronti, G. Caliano, R. Carotenuto, A. Savoia, M. Pappalardo, E. Cianci, and V. Foglietti, "Capacitive micromachined ultrasonic transducer (CMUT) arrays for medical imaging," Microelectronics Journal, vol. 37, no. 8, pp. 770-777, Aug. 2006, issn: 0026-2692. doi: 10.1016/j.mejo.2005.10.012. [Online]. Available: http: / / www. sciencedirect. com/science/article/pii / S0026269205003812 (visited on 04/09/2018).

[8] S. Zhou and J. A. Hossack, "Reducing inter-element acoustic crosstalk in capacitive micromachined ultrasound transducers," IEEE Transactions on Ultrasonics, Ferroelectrics, and Frequency Control, vol. 54, no. 6, pp. 1217-1228, Jun. 2007, issn: 0885-3010. doi: 10.1109/TUFFC.2007.375.

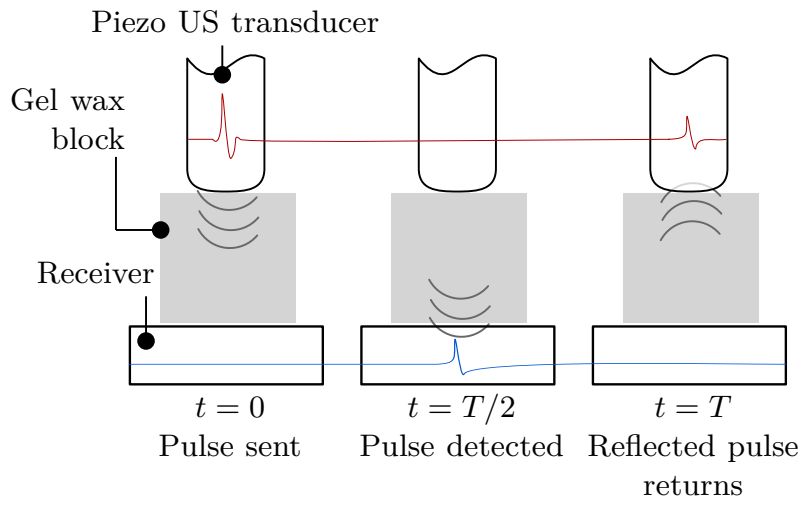

Fig. 3. To demonstrate the device, the signals from the piezo transducer and the receiver are separated in the time domain. The red trace represents the signal from the transducer, and the blue trace the signal from the receiver

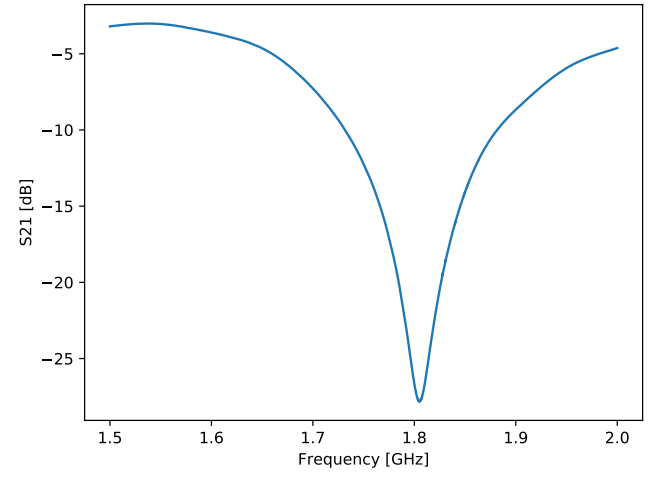

Fig. 4. The transmission spectrum $\left(S_{21}\right)$ of the device, showing a resonance at $1.8 \mathrm{GHz}$

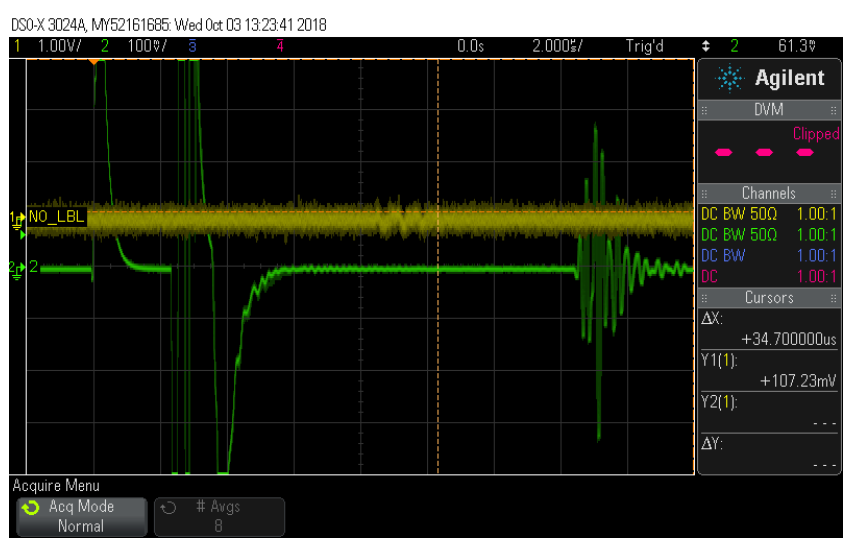

Fig. 5. Oscilloscope trace of the received ultrasound signal (yellow) and the pulse/echo response from the transducer (green)

[9] E. Maneas, W. Xia, O. Ogunlade, M. Fonseca, D. I. Nikitichev, A. L. David, S. J. West, S. Ourselin, J. C. Hebden, T. Vercauteren, and A. E. Desjardins, "Gel wax-based tissue-mimicking phantoms for multispectral photoacoustic imaging," EN, Biomedical Optics Express, vol. 9, no. 3, pp. 1151-1163, Mar. 2018, issn: 2156-7085. doi: 10.1364/BOE.9.001151. [Online]. Available: https://www.osapublishing.org/ boe / abstract . cfm ? uri = boe- $9-3-1151$ (visited on 10/04/2018). 